\title{
The Skyrocketing rate of Cesarean section in Egypt
}

\author{
Mohamed Kandil* \\ Professor of Obstetrics and Gynecology, Faculty of Medicine, Menoufia University, Shibin Elkom-Menoufia, Egypt
}

\begin{abstract}
Cesarean section rates have been steadily increasing in Egypt from a low of 4.6\% in 1992, 6.7\% in 1995, $10.3 \%$ in 2000 , to about 52\% in 2014. In an attempt to explain this steady rise, health professionals mostly blame mothers claiming that the leading factor is the increasing rate of Cesarean section on request. I believe the skyrocketing rates are due to a mixture of reasons related to mothers, obstetricians and the environment where they practice Obstetrics.
\end{abstract}

Cesarean section is now the most commonly performed surgery in the world. A recent study documented that Egypt ranked 3rd among world countries with an estimated rate of Cesarean section of $51.8 \%$ [1].

This skyrocketing rate is probably related to a mixture of reasons. The increased maternal request for Cesarean section especially in primigravidae is one reason. Women are afraid of the associated pain and long hours of labor and delivery. A story of a prolonged, obstructed or instrumental labor from a parturient can be a panic to other pregnant women in her neighborhood. The media, specifically TV series and movies, are publicizing very untruthful images about normal delivery and its consequences.

Obstetricians are also responsible for the high rates of Cesarean in Egypt. They may claim superiority and safety of Cesarean section over normal delivery on the long-term overall health in the absence of evidence. Cesarean section on request is more convenient to obstetrician as it allows him/her to arrange life without night calls or disturbing a vacation schedule. It is quite clear that rates of Cesarean section markedly increase during summer compared to winter time and in the weekdays compared to weekends (unpublished data). In addition, the revenue is much higher than that for a normal delivery. Obstetricians are therefore reluctant to properly counsel their patients about the advantages of normal labor versus Cesarean section. This is unethical.

Thirdly, the fear of litigations contributes to the situation. There is increased awareness in the Egyptian society with the medical profession and malpractice issues. Attorneys are trying to make use of the current pressing economic circumstances and offering their services for a percent of a possible compensation. In response to this attack, Egyptian obstetricians started to practice defensive medicine. A clearly evident example for this type of medicine is the marked avoidance of VBAC which is universally recommended $[2,3]$.

Finally, it the author's impression that there is a problem with the regulations governing the local medical practice in Egypt. The private sector needs major adjustments. It is the routine that the obstetrician who cares for a woman's pregnancy is the one who will deliver her. We are moving toward a medically insured health system and in such, a woman should be able to choose the obstetrician who will take care of her pregnancy and to choose a hospital where qualified doctors are available all around the clock to deliver in. The choice should be between where she is going to deliver and not who will deliver her. This will overcome the effect of having Cesareans performed for a one obstetrician's convenience. In addition, we need a legal platform to clearly define a medical complication and a medical malpractice and the responsibilities of all personnel operating in the medical profession. This will make obstetricians feel comfortable doing their job implementing proper guidelines without fears of being sued because of absent legal regulations.

\section{References}

1. Egypt demographic and Health Survey report (2014)

2. The American college of Obstetrics and Gynecology (2017) ACOG Releases New Guidance Aimed at Making VBAC Available to More Women.

3. The Royal College of Obstetricians and Gynecologists (2015) Birth after previous Cesarean birth. Green-top Guideline No. 45.
Copyright: (C)2018 Kandil M. This is an open-access article distributed under the terms of the Creative Commons Attribution License, which permits unrestricted use, distribution, and reproduction in any medium, provided the original author and source are credited.
${ }^{\star}$ Correspondence to: Mohamed Kandil, Professor of Obstetrics and Gynecology, Faculty of Medicine, Menoufia University, Shibin Elkom-Menoufia, Egypt, E-mail: Mohamed.kandeel@med.menofia.edu.eg

Key words: cesarean on request, on demand cesarean, cesarean litigations

Received: July 25, 2018; Accepted: August 07, 2018; Published: August 10, 2018 